19 Revue d'histoire du XIXe siècle

Société d'histoire de la révolution de 1848 et des

révolutions du XIXe siècle

25 | 2002

Le temps et les historiens

\title{
La gloire et le temps
}

\section{Corinne Legoy}

URL : http://journals.openedition.org/rh19/434

DOI : 10.4000/rh19.434

ISSN : $1777-5329$

\section{Éditeur}

La Société de 1848

\section{Édition imprimée}

Date de publication : 1 décembre 2002

Pagination : 165-170

ISSN : 1265-1354

Référence électronique

Corinne Legoy, "La gloire et le temps », Revue d'histoire du XIXe siècle [En ligne], 25 | 2002, mis en ligne le 29 juin 2005, consulté le 03 mai 2019. URL : http://journals.openedition.org/rh19/434 ; DOI :

$10.4000 /$ rh19.434

Ce document a été généré automatiquement le 3 mai 2019.

Tous droits réservés 


\title{
La gloire et le temps
}

\author{
Corinne Legoy
}

1 Tout voué à produire du mythe, l'éloge tend, par essence, à l'intemporalité, fondement de la gloire qu'il consacre; dans l'écriture même de l'éloge, la tension des-historicisante est donc une constante, qui réduit l'histoire à des exempla et ses acteurs à des archétypes. Scandant le temps de la monarchie selon une mécanique fixée au moins depuis le XVI ${ }^{\mathrm{e}}$ siècle ${ }^{1}$, il semble de surcroit figé dans une vénération respectueuse et sacralisante. Pourtant, l'éloge sourd bien de l'événement qu'il célèbre ou déplore et s'apparente aussi à une écriture réactive, en prise sur le temps politique. Dans la production thuriféraire de la Restauration, ce paradoxe est patent d'une écriture en tension, prise entre l'événement qui la suscite et la représentation idéalisante qu'elle vise. Écriture qui tisse une trame événementielle particulière, faite d'évocations, de parallèles et de transpositions, où la surimposition des temps est systématique, le présent s'arrimant au passé et servant l'avenir. L'éloge apparaît ainsi contraint simultanément par un rêve d'arrachement au temps humain ${ }^{2}$ et par un impératif de contemporanéité; tout comme l'oraison funèbre, et pour reprendre les termes de Nicole Loraux, il se caractérise par "l'inextricable enchevêtrement du temps vécu de l'histoire [...] et d'une autre temporalité, celle de la célébration, qui vise à faire du maintenant un toujours" ${ }^{3}$. Ce faisant, les plus de 1500 poèmes écrits entre 1815 et 1830 pour célébrer les Bourbons ${ }^{4}$ reconstruisent une temporalité politique et historique, qui leur est propre, faite de tris partisans, de mises en sourdine et de mises en exergue. Les représentations du temps dans l'éloge trahissent en effet nettement une perception hypertrophiée et lacunaire, affective et magique, d'un passé et d'un présent à la fois sacralisés et instrumentalisés ${ }^{5}$. Mise en scène d'un temps affectif, fait de surimpositions et de crispations ; élaboration d'un temps mythique, livré sur le mode du "quale" ${ }^{6}$ à la fois indifférencié et manichéen, orienté et signifiant, didactique et épiphanique. La logique est celle d'un discours qui retient et structure "ce qui est exemplaire" ", à la charnière des contraintes rhétoriques et des tensions mémorielles de la Restauration.

2 À l'origine de tout éloge se trouve un événement --fête rituelle de la monarchie restaurée, heure de gloire ou grand drame ${ }^{8-}$ auquel le texte vient répondre dans l'instant. La 
proclamation de la concomitance du geste d'écriture et de l'événement, faisant de la louange une traduction immédiate du ressenti, expression sans décalage de l'expérience de l'instant, est la caractéristique de ce discours. Tous les événements originaires ${ }^{9}$ sont en effet l'objet d'une actualisation, qui abolit la notion de passé proche au profit des représentations d'un présent toujours en acte, vécu et sensible. Usage récurrent du présent, rhétorique performative et postures de la surprise logent efficacement l'instant vécu dans le creux de la parole de gloire et par là-même "présentifient" l'événement. Ces représentations de l'actualité qui font fi des décalages temporels relèvent de la mise en scène d'un temps affectif; de fait, les registres d'évocation de l'événement arrachent tout le temps raconté à l'indifférence et reposent sur la coprésence (discursive) de l'expérience passée et de l'expérience actuelle. Cette mise en scène émotionnelle est particulièrement nette dans les évocations de l'assassinat du duc de Berry, toutes tendues vers la reviviscence émue et émouvante. Ainsi un Canela déroule-t-il la scène comme si, témoin actif, il avait le pouvoir d'inverser le cours des événements. Il joue d'un feint suspens, qui rend incertaine l'issue de la scène. Le poète voit Berry et le poignard de l'assassin, il met en garde le duc, implore : qu'il voie le poignard, qu'il l'arrête... La prière du poète est cependant inutile puisque l'assassin frappe : c'en est fait ${ }^{10}$. Signifiantes aussi sont les fictions de l'imprévu et les mises en scène de la surprise de l'auteur. Elles présentent généralement la même structure: tout d'abord, l'auteur est saisi par un trouble intense, souvent suscité par des cris, des pleurs ou une apparition qui le frappent, puis, sous le coup de l'émotion, il s'interroge vivement et décrypte peu à peu l'événement déclencheur. Par son actualisation sur le mode interrogatif, elles axent ainsi l'évocation de l'événement sur les émois qu'il suscite. Citons, par exemple, les mots d'Antoinette Robert, prélude à son cri de joie à la naissance du duc de Bordeaux: "Quelle douce mélodie se fait entendre ! D'où partent ces sons harmonieux qui troublent le silence de la nuit ? L'air chéri des Français s'élève jusqu'aux nues... Le grand Henri a-t-il quitté la voûte céleste ? Vient-il annoncer à la France qu'il va naître un autre Henri ? ... Écoutons : déjà mille échos répètent un Prince ! ... un Bourbon !..." ${ }^{11}$.

3 Toutes les représentations du présent dans l'éloge sont donc marquées par cette fiction d'immédiateté, de réception de l'instant dans le feu de l'urgence et de l'émotion. Cependant, si les thuriféraires cherchent à entretenir une mémoire vive de l'événement dans la radicalité de son surgissement et de son écho affectif, paradoxalement, ils l'excèdent aussi et le brouillent en greffant sur lui tout un passé révolutionnaire sans cesse réactivé. De fait, la Révolution française est le moment obsessionnel des éloges de la Restauration et marque les représentations de tous les événements politiques de 1815 à 1830. Ainsi l'assassinat du duc de Berry est-il cet autre régicide qui fait planer de nouveau l'ombre révolutionnaire ${ }^{12}$; ainsi la révolte espagnole contre Ferdinand VII est-elle l'œuvre de la "horde furieuse" qui sévit en France ${ }^{13}$ de même que l'opposition politique, indistinctement, incarne une hydre révolutionnaire éternellement menaçante. Dans cette invariable identification de l'événement nouveau à la Révolution française, dans cette dilution traumatique de la frontière passé-présent, la littérature d'éloge du premier XIX ${ }^{\mathrm{e}}$ siècle se révèle comme une écriture de la phobie.

4 Ce temps affectif, pour finir sur ce point, est aussi marqué par la surimposition des couches temporelles, la célébration --ou la déploration-- du présent réactivant invariablement un bonheur ou un malheur antérieur. La célébration du mariage du duc de Berry, par exemple, alimente celle du retour des Bourbons ${ }^{14}$, la victoire en Espagne est l'occasion d'une nouvelle célébration de la naissance du duc de Bordeaux ${ }^{15}$, quand 
l'insurrection en elle-même avait ravivé le souvenir du 13 février $1820{ }^{16}$. Ces procédures discursives de commémoration semblent là encore placées sous le signe de la hantise, le redoublement de l'intensité du présent par l'évocation d'une émotion passée fonctionnant comme une mécanique tantôt conjuratoire --pour le mariage du duc de Berry par exemple--, tantôt mobilisatrice et politique-- pour la mort du duc de Berry ou l'intervention en Espagne, en particulier.

Indissociable de ces procédés d'élaboration et de mise en scène d'un temps affectif : la construction d'un temps mythique. La temporalité de la célébration est bien en effet celle du mythe, où le passé est hypertrophié, l'avenir conjuré et l'histoire abolie.

Dans l'éloge, le passé lointain est un passé monumental et schématique, constamment évoqué sur le mode de l'hyperbole. Sont opposés, en une distinction manichéenne et radicale, temps merveilleux et temps obscurs, âge d'or monarchique et apocalypse révolutionnaire. La lecture est à la fois qualitative et globalisante : la Révolution française des panégyristes est un temps sans dates ni faits, une longue et indistincte succession de maux, "temps malheureux de deuil, de trouble et d'anarchie" ${ }^{17}$, années ingrates de maux et de souffrances ${ }^{18}$ ou "cercle immense de calamités" ${ }^{19}$. Ce regard est d'ailleurs celui de toute la tradition contre-révolutionnaire qui ignore les idées d'évolution et de radicalisation. D'un côté, donc, se trouve le passé idyllique des "temps jadis", passé monarchique lointain et sans véritables repères, de type plus féodal qu'Ancien Régime. Les louanges célèbrent à loisir ces temps glorieux ${ }^{20}$; temps de l'ordre et de la paix, temps sans troubles ni débats, sans jalousie vis-à-vis des grands ${ }^{21}$; temps des bonnes mœurs et des bons aïeux. De l'autre, on a le passé infernal des "temps d'égarement", temps révolutionnaires qu'il faut oublier, déplorer ou expier, époque d'esclavage, de guerres et de morts, de subversion de toutes les valeurs et de perversion ${ }^{22}$.

7 Dans ce registre mythique, où le temps est pensé en termes manichéens et qualitatifs, l'avenir est à la fois constamment évoqué sur le mode d'une profération incantatoire et messianique et instamment conjuré. Deux modalités essentielles de la conjuration se dégagent de l'ensemble des éloges : soit l'avenir est conçu comme la réitération sublimée d'un passé idéalisé, soit il est conçu comme un étirement radieux du présent. La naissance du duc de Bordeaux, nouvel Henri IV, laisse ainsi présager le retour d'un règne bienfaisant pour les Français. Il saura, comme le Béarnais, se battre, être franc-buveur et vert-galant et il égalera son courage : "nous aurons notre Henri / Ta valeur nous rendra, si une ligue ennemie ose se manifester, les beaux jours d'Arque et d'Ivry" ${ }^{23}$. Un peu différemment le présent peut, lui aussi, être étiré en un futur rassurant : le second retour de Louis XVIII en 1815, par exemple, ouvre pour les panégyristes une ère de bonheur destinée à durer toujours, l'avenir se muant en une éternité radieuse. S'élabore ainsi un temps mythique, caractérisé par la dilution de la succession temporelle et par la réduction de l'incertitude ou de l'inexplicable, présent et avenir étant commandés par un passé idéal. Nouveauté, rupture, progrès ou révolution sont donc des notions absolument étrangères à la parole de gloire, qui réduit chaque événement à un passé connu. On constate, par exemple, une dilution de la singularité de la Révolution elle-même par son identification aux guerres de religion ou par son assimilation à une résurgence ligueuse, vaincue par Dieu et le Roi. De la même façon, l'assassinat du duc de Berry est interprété selon une imagerie qui servit en son temps à rendre compte de l'attentat de Damiens, quand les insurgés espagnols sont, invariablement, de nouveaux Clément ou d'autres Louvel. Par cette réduction mimétique du présent au passé, la parole de gloire opère un aplanissement du temps historique, qui se trouve renforcé par le recours à une causalité 
historique figée ou simpliste --lecture manichéenne de l'histoire, produit du conflit entre le Bien et le Mal, entre Dieu et les forces sataniques ; lecture providentialiste, où elle est le fruit d'une transcendance tantôt vengeresse, tantôt bienveillante.

Dans ce temps mythique, l'histoire est donc abolie, au profit d'un écoulement temporel marqué par la continuité et la répétition. La vision est nettement conservatrice qui assigne au temps un déroulement itératif, fait du passé un modèle et non une étape préparatoire à dépasser. L'âge d'or perdu est donc destiné à revenir, la Restauration renoue avec des temps idylliques, les Français redeviennent ces Français galants des temps jadis et le Roi lui-même est un monarque d'autrefois. Le présent est toujours l'image d'un passé merveilleux, et l'avenir lui-même redonnera à la France, parfois mieux encore que le présent, sa gloire d'antan. Cette lecture de l'histoire, qui fond le passé, le présent et l'avenir en une continuité rassurante nous renvoie à la culture légitimiste en même temps qu'il est un procédé rhétorique : il glorifie le pouvoir, le légitime et l'inscrit dans la longue durée capétienne. Si ces lectures du passé et des événements contemporains se fondent sur des interprétations de l'histoire, on ne saurait cependant y voir une pensée cohérente du devenir historique. Certes, elles sont informées par toute une chronosophie chrétienne --reprise par la pensée contre-révolutionnaire--, mais elles se situent en fait à la fois en-deçà et au-delà : elles articulent différents systèmes de représentations du temps historique (qui oscille ainsi entre temps cyclique et temps linéaire ou cumulatif) et différentes interprétations de l'histoire (marquées autant par la pensée contre-révolutionnaire, que par l'illuminisme, les écrits d'un Barruel ou les publicistes ultras). La pauvreté intellectuelle des schémas explicatifs de l'éloge, d'ailleurs souvent contradictoires ou suraccumulés, semble témoigner d'un décrochement entre l'histoire et certains cadres de pensée impuissants à la saisir autrement qu'en la figeant, disjonction certaine entre le temps lent et long des représentations et ce temps bouleversé de la charnière du XVIII ${ }^{e}$ et du XIX ${ }^{e}$ siècles. Quoi qu'il en soit, la parole de gloire s'impose nettement comme un ensemble de lectures irraisonnées de l'histoire, affectivement, théologiquement et idéologiquement construites.

9 Ce temps façonné par la parole de gloire est donc un temps monumental et sans épaisseur : la succession temporelle est diluée, le passé commande le présent et l'avenir, l'histoire est abolie au profit d'une continuité-éternité rassurante. Pourtant les éloges portent aussi les stigmates de l'événement : passé révolutionnaire et présent politique de la Restauration alimentent un discours de la hantise qui rêve de conjurer les aléas de l'histoire, bien plus qu'il n'aspire à célébrer le temps monarchique.

Corinne Legoy est doctorante à l'Université Paris 1-Panthéon-Sorbonn

\section{NOTES}

1.. À partir de François $\mathrm{I}^{\mathrm{er}}$, le genre de l'éloge royal se développe considérablement, pour trouver son apogée sous Louis XIV. On peut consulter sur ce sujet Giovanni DOTOLI [dir.], Politique et littérature en France aux XVI et XVII siècles, Paris, Éditions Didier Érudition, 1997, 579 p. ; Nicole FERRIER-CAVERIVIÈRE, L'Image de Louis XIV dans la littérature française, 
Paris, Presses universitaires de France, 1981, 439 p. ; Françoise BARDON, Le Portrait mythologique à la cour de France sous Henri IV et Louis XIII. Mythologie et politique, Paris, Éditions Picard, 1974, 326 p.

2.. À la différence de la littérature d'engagement, voir Benoît DENIS, Littérature et engagement. De Pascal à Sartre, Paris, Éditions du Seuil, 2000, chapitre 2, pp. 30-42.

3.. Nicole LORAUX, L'Invention d'Athènes. Histoire de l'oraison funèbre dans la cité "classique", Paris, Éditions de l'École des hautes études en sciences sociales, 1981. pp. 13-14.

4.. L'étude de ce corpus a été menée dans le cadre d'une thèse en cours de rédaction : Corinne LEGOY, La louange et la célébration des Bourbons sous la Restauration, thèse d'histoire sous la direction d'Alain Corbin, Université Paris 1-Panthéon-Sorbonne. Le corpus englobe la quasi-totalité des éloges, manuscrits ou imprimés, écrits entre 1815 et 1830, à la gloire des Bourbons restaurés. L'approche choisie est résolument protéiforme puisque l'éloge est analysé à la fois comme une pratique culturelle et politique et comme une écriture polymorphe. Aussi le corpus mêle-t-il les genres et les formes pour cerner "la parole de gloire" : odes, épîtres, épithalames... mais aussi chansons (couplets, rondes et cantates. 5.. Voir, pour la distinction histoire-mémoire, Pierre NORA, "Entre Mémoire et Histoire. La problématique des Lieux", dans Pierre NORA [dir.], Les Lieux de Mémoire, Quarto, Paris, Éditions Gallimard, 1997, tome 1. On peut lire aussi, sur cette question, Jacques LE GOFF, Histoire et mémoire, Paris, Éditions Gallimard, 1988.

6. Max MILNER, "Temps historique et temps mythique dans le romantisme français", dans Mélanges littéraires François Germain, Dijon, 1979, pp. 109-124. Max Milner citant Cassirer : "La vision mythique et religieuse du monde, en revanche, n'admet jamais que le temps devienne un quantum homogène. Le temps lui est au contraire donné, quelque universelle que puisse être finalement la figure qu'elle attribue, sous la forme spécifique d'un "quale"“; idem, p. 112.

7.. On transpose ici la différence dégagée par Michel de Certeau entre l'écriture historique et l'écriture hagiographique : "la combinaison des actes, des lieux et des thèmes indique une structure propre qui se réfère non pas essentiellement à "ce qui s'est passé", comme le fait l'histoire, mais à "ce qui est exemplaire"“", Michel DE CERTEAU, L'écriture de l'histoire, Paris, Éditions Gallimard, 1975, p. 275.

8. Citons seulement les principaux : outre les fêtes royales (saint Louis le 25 août et saint Charles le 4 novembre), ce sont le second retour des Bourbons en 1815, le mariage du duc de Berry en 1816, l'évacuation du territoire par les Alliés en 1818, l'assassinat du duc de Berry et la naissance du duc de Bordeaux en 1820, l'intervention en Espagne en 1823, la mort de Louis XVIII en 1824 et le sacre de Charles X en 1825.

9.. Nous appelons ainsi les événements qui servent de prétexte à l'écriture d'un éloge, soit l'objet premier de la parole de gloire, mentionné invariablement dans les titres.

10.. CANELA, Ode sur la mort de son Altesse Royale Mgr le duc de Berry, Blois, Imprimerie de Verdier, 1820.

11.. Antoinette ROBERT, Cri de joie d'une Française sur la naissance de son Altesse Royale Mgr le duc de Bordeaux, par Imprimerie de Mme Ve Porthmann, Paris, 1820 ; on peut citer aussi, seul exemple d'un procédé récurrent dans les textes sur l'assassinat du duc de Berry : “Où court ce peuple errant dans cette nuit profonde? / Quel est ce meurtrier ? quel est cette victime ? / Sur cette épouse en pleurs quel sang a rejailli ?" ; voir Charles LOYSON, Ode sur l'attentat du 13 février 1820, Paris, Imprimerie de Denugon, 1820.

12.. Citons seulement, parmi une foule d'exemples, A. D'EGVILLY, Nuits françaises, sur l'attentat du 13 février 1820, suivies d'une élégie sur la mort de son Altesse Royale Mgr le duc de Berri, Paris, Imprimerie d'A. Boucher, 1820, dans lesquelles l'auteur souligne que la France 
croit être revenue aux temps de terreur ; et une anonyme Ode sur la mort de son Altesse Royale Mgr le duc de Berry, Paris, Imprimerie de Le Normant, 1820, pour ces vers : "Ce sang tracera ton histoire : / Déjà s'attache à ta mémoire / Une horrible calamité ; / Providence du régicide, / Au poignard sanglant d'un séide / Tu devras ta célébrité !".

13.. Ainsi Philippe Albert place-t-il la révolte espagnole "dans la lignée de l'assassinat du duc de Berry, second régicide" et souligne que "cette horde impure", méditant de nouveaux attentats, trouva un refuge en Espagne, "la terre de l'honneur"; Philippe ALBERT, L'Espagne délivrée, Paris, Imprimerie A. Brossier, 1820. De la même façon, PevrieuLassale, souligne que la lutte en Espagne contre les insurgés est un combat contre "la ligue", "du trône et de l'autel odieuse ennemie", responsable de la Révolution en France ; voir PEVRIEU-LASSALE, L’Espagne délivrée, poème, Toulouse, Imprimerie de Bénichet aîné, 1820.

14.. Voir, entre autres exemples, VALANT, Épithalame sur le mariage de son Altesse Royale Mgr le duc de Berry et de la princesse Marie-Caroline de Naples, Paris, Imprimerie de Ballard, 1816.

15.. Ainsi dans La Guerre d'Espagne, poème en stances régulières; ou Bouquet au Roi, pour la fête de Sa Majesté, 25 août 1823, Paris, Imprimerie d'A. Boucher, 1823 ; ou dans BOUTEREAU, La Guerre d'Espagne, élégie, Paris, Imprimerie de A. Boucher, 1823.

16.. Voir, notamment, LETOURNAN, La Guerre d'Espagne, Paris, Imprimerie de Pillet aîné, 1823.

17.. Églogue ou entretiens d'un père avec son fils sur les événements politiques actuels, par un citoyen d'Avignon, S.l., 1815.

18.. M. C. ADRIEN LE CLERE, Le Retour de Buonaparte, Épittre au Roi, Paris, 1815.

19.. A. LEGRAND, Le Retour des Bourbons, Étrennes aux Français, Paris, Imprimerie Testu, 1815.

20.. Frédéric de BERMONDET DE CROMIÈRES, "Rappelle-toi ces temps fameux vantés dans l'histoire pour toujours", Chant français, dans Frédéric de BERMONDET DE CROMIÈRES, Recueil de couplets composés et chantés en l'honneur de Sa Majesté, Paris, Imprimerie de Dondey-Dupré, 1825.

21.. Églogue ou Entretiens d'un père avec son fils, sur les événemens politiques actuels, par un citoyen d'Avignon, S.l, 1815.

22.. On peut citer ici X.-V. DRAP-ARNAUD, Ode au peuple français sur les malheurs de l'anarchie et de l'ambition, à l'occasion de l'avènement légitime du roi Charles $X$ au trône de France , Paris, Imprimerie de C.-J. Trouvé, 1824, ainsi que A. LEGRAND, Le Retour des Bourbons, Étrennes aux Français, Paris, Imp. de Testu, 1815.

23.. LAUMIER, Ode sur la mort de son Altesse Royale Mgr le duc de Berry et la naissance de son Altesse Royale Mgr le duc de Bordeaux, Paris, Imprimerie de Sétier, 1820. 